\title{
A Simple Proof of the Alternation Theorem
}

\author{
P. P. Vaidyanathan \\ Dept. of Electrical Engineering \\ California Institute of Technology \\ Pasadena, CA 91125 \\ Email: ppvnath@systems.caltech.edu
}

\author{
T. Q. Nguyen \\ Dept. of Electrical and Computer Engr. \\ University of California \\ San Diego, CA 92093 \\ Email: nguyent@ece.ucsd.edu
}

\begin{abstract}
A simple proof of the alternation theorem for minimax FIR filter design is presented in this paper. It requires no background on mathematical optimization theory, and is based on easily understood properties of filters with equiripple behavior. The method is similar to the classical counting argument used in early mathematics literature. The contribution here is a simplified presentation which directly uses filter design language. ${ }^{1}$
\end{abstract}

\section{INTRODUCTION}

The McClellan-Parks method based on the Remez exchange algorithm has been used for the design of equiripple FIR filters for more than thirty years [1], [3]-[8]. A discussion of the method is included in nearly all well-known signal processing texts. The algorithm is based on a result called the alternation theorem which gives a set of conditions under which a filter design is optimal in the minimax sense. This theorem is therefore at the heart of the method. In signal processing texts this theorem is ususally not proved, but a reference is given to mathematical optimization texts such as [2]. The purpose of this paper is to provide a simple proof which can be presented in the classroom and requires no background on mathematical optimization theory. The argument will be based on the simple fact that a polynomial of order $M$ cannot have more than $M$ zeros. Similar proofs based on counting arguments were presented in old fashioned mathematics literature (e.g., [9] pages 56 and 61-62). The main contribution in this paper is to present a proof directly in filter-design language in a way that is simple and accessible to readers with introductory signal processing background.

First some preliminaries. All discussions are restricted to the case of linear phase real coefficient FIR filters. It is well known [6], [8] that there are four types of such filters. Type 1 filters have the form

$$
H(z)=\sum_{n=0}^{N} h(n) z^{-n},
$$

where $N$ is even and

$$
h(n)=h(N-n)
$$

${ }^{1}$ Work supported in parts by the National Science Foundation grant CCF-0428326 and the California Institute of Technology. so that $[6],[8]$

$$
H\left(e^{j \omega}\right)=e^{-j \omega M} \sum_{n=0}^{M} b_{n} \cos (\omega n)
$$

where $M=N / 2$. The factor $e^{-j \omega M}$ representing the linear phase part will be ignored in all discussions. Since Type 2,3 , and 4 filters can be expressed in terms of Type 1 filters [8], the theory and design of linear phase filters is centered around the design of the coefficients $\left\{b_{n}\right\}$ in the sum $\sum_{n=0}^{M} b_{n} \cos (\omega n)$. This summation is used to approximate a real desired response $D(\omega)$ in $0 \leq \omega \leq \pi$ with a specified weighting function $W(\omega)>0$ on the error. The approximation error is

$$
E(\omega)=D(\omega)-\sum_{n=0}^{M} b_{n} \cos (\omega n)
$$

whereas the weighted error of approximation is defined by

$$
\widehat{E}_{b}(\omega)=W(\omega)[D(\omega)-\underbrace{\sum_{n=0}^{M} b_{n} \cos (\omega n)}_{\text {call this } B(\omega)}]
$$

Observe that $W(\omega)$ and $D(\omega)$ are defined in the union $\mathcal{F}$ of all bands of interest (passbands and stopbands) so that $\mathcal{F} \subset[0, \pi]$. Figure 1 shows an example of a three band approximation problem, demonstrating the various quantities involved. More generally $D(\omega)$ and $W(\omega)>0$ can be arbitrary, not necessarily piecewise constants. The alternation theorem gives the conditions under which the maximum of the weighted error $\widehat{E}(\omega)$ in $\mathcal{F}$ is minimized. (The subscript $b$ in $\widehat{E}_{b}(\omega)$ is dropped here for simplicity; it will be required later in the proof for clarity).

The alternation theorem. Let $\mathcal{F}$ denote the union of closed intervals (frequency bands of interest) in $0 \leq \omega \leq \pi$ where a real desired response $D(\omega)$ and a positive weighting function $W(\omega)$ have been specified. The function

$$
B(\omega)=\sum_{n=0}^{M} b_{n} \cos (\omega n)
$$


is the unique weighted minimax approximation of $D(\omega)$ in $\mathcal{F}$ with respect to the weighting function $W(\omega)$ (i.e., $B(\omega)$ minimizes the peak weighted-error $|\widehat{E}(\omega)|$ in $\mathcal{F})$ if and only if there exist at least $M+2$ distinct frequencies

$$
\omega_{1}<\omega_{2} \ldots<\omega_{M+2}
$$

(called extremal frequencies) in the set $\mathcal{F}$, satisfying two properties:

1) The weighted error $\widehat{E}(\omega)$ alternates, that is,

$$
\widehat{E}\left(\omega_{1}\right)=-\widehat{E}\left(\omega_{2}\right)=\widehat{E}\left(\omega_{3}\right)=\ldots
$$

2) the maximum of $|\widehat{E}(\omega)|$ is attained at each of these frequencies, that is,

$$
\max _{\omega \in \mathcal{F}}|\widehat{E}(\omega)|=\left|\widehat{E}\left(\omega_{k}\right)\right|
$$

for all $k . \diamond$

$D(\omega)$

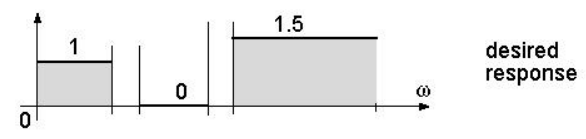

$W(\omega)$

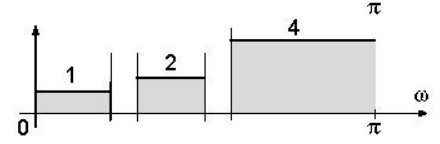

weight

$E(\omega)$

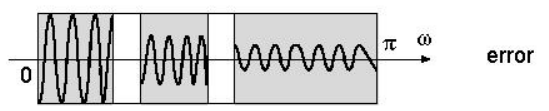

$\widehat{\mathrm{E}}_{\mathrm{b}}(\omega)$

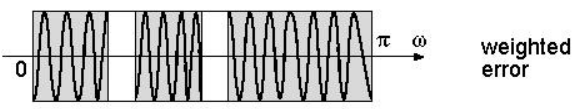

$B(\omega)$

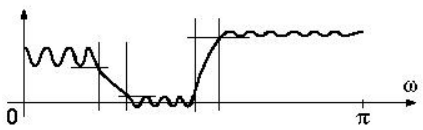

approximation

Fig. 1. Example of a desired response $D(\omega)$, weighting function $W(\omega)$, approximation errro $E(\omega)=D(\omega)-B(\omega)$, weighted error $\widehat{E}_{b}(\omega)$, and the approximation $B(\omega)$.

\section{PRoOF of THE ALTERnAtion THEOREM}

The existence of the solution $B(\omega)$ follows from compactness arguments (e.g., see [9], page 23) and will not be addressed here. Assuming existence, we show that the conditions in the theorem ensure that $B\left(e^{j \omega}\right)$ is the unique optimal solution. Assume then that $B(\omega)$ is the weighted equiripple solution with at least $M+2$ extremal frequencies.
So the weighted error $\widehat{E}_{b}(\omega)$ in Eq. (1) is equiripple, and fits inside the shaded boxes as demonstrated in Fig. 2(a) (each shaded box is a desired passband or stopband). Let there be $N_{k}$ extrema inside a given shaded box so that the total number of extremal frequencies is

$$
N_{1}+N_{2}+\ldots+N_{K}
$$

where $K$ is the number of bands. For example, in Fig. 2(a) we have $K=3$ and

$$
N_{1}=8, \quad N_{2}=9, \quad N_{3}=14
$$
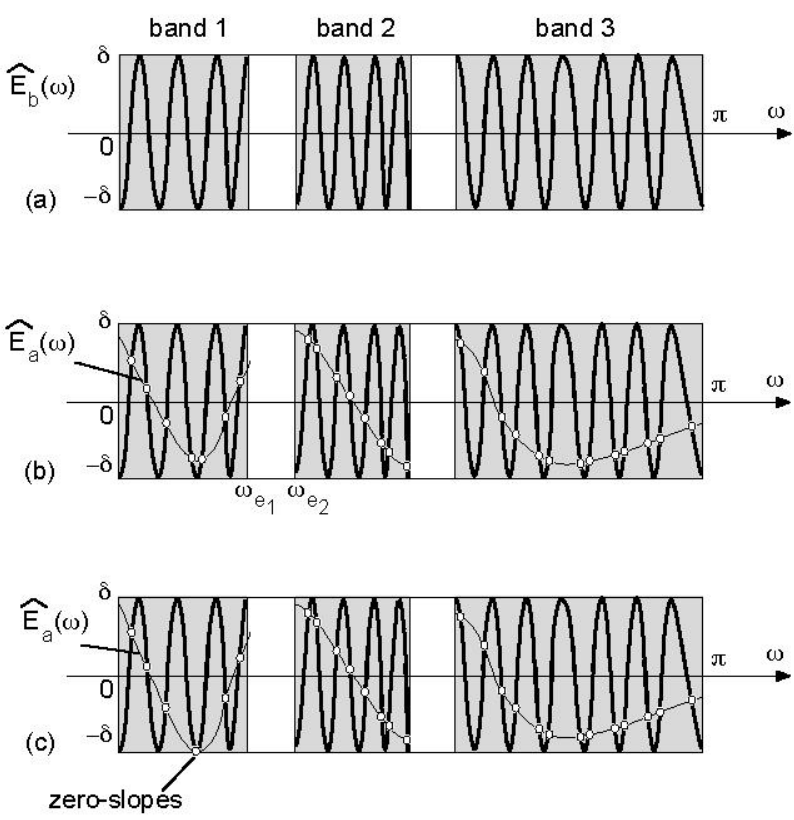

Fig. 2. (a) Example of a weighted error function $\widehat{E}_{b}(\omega)$ with equiripple behavior, (b), (c) examples of other weighted errors $\widehat{E}_{a}(\omega)$ which can be fitted into the shaded boxes. See text.

If the necessary condition of the alternation theorem is satisfied, we have at least $M+2$ extrema, that is,

$$
\sum_{k=1}^{K} N_{k} \geq M+2
$$

Now consider another approximation of the same order

$$
A(\omega)=\sum_{n=0}^{M} a_{n} \cos (n \omega)
$$


such that its peak error is at least as small as in the equiripple case. That is, the weighted error

$$
\widehat{E}_{a}(\omega)=W(\omega)[D(\omega)-\underbrace{\sum_{n=0}^{M} a_{n} \cos (n \omega)}_{A(\omega)}]
$$

satisfies

$$
\max _{\omega \in \mathcal{F}}\left|\widehat{E}_{a}(\omega)\right| \leq \max _{\omega \in \mathcal{F}}\left|\widehat{E}_{b}(\omega)\right|
$$

This means that $\widehat{E}_{a}(\omega)$ also fits the shaded boxes as demonstrated by the thin curve in Fig. 2(b). We will show then that

$$
A(\omega)-B(\omega) \equiv 0,
$$

that is, the two approximations are one and the same! The argument will be based on the simple fact that a polynomial of order $M$ has no more than $M$ zeros. First observe that since $\widehat{E}_{b}(\omega)$ swings between the two extreme values $\delta$ and $-\delta$, the plot of $\widehat{E}_{a}(\omega)$ intersects the equiripple plot $\widehat{E}_{b}(\omega)$ at least $N_{k}-1$ times in the $k$ th band. That is, the difference

$$
\Delta \widehat{E}(\omega) \triangleq \widehat{E}_{b}(\omega)-\widehat{E}_{a}(\omega)
$$

has at least $N_{k}-1$ zeros in the $k$ th band. For example the number of intersections in Fig. 2(b) are 7,8, and 13. Letting $N_{z}$ be the number of zeros of $\Delta \widehat{E}(\omega)$ in $0 \leq \omega \leq \pi$, we therefore have

$$
N_{z} \geq \sum_{k=1}^{K} N_{k}-K .
$$

The careful reader will notice that it is possible that an extremum of $\widehat{E}_{a}(\omega)$ coincides with that of $\widehat{E}_{b}(\omega)$ in which case the number of intersections in a band can be fewer than $N_{k}-1$ as demonstrated in the leftmost band of Fig. 2(c). Since both error curves have zero slopes at such a point, the difference $\Delta \widehat{E}(\omega)$ has zero slope there. That is, the zero of $\Delta \widehat{E}(\omega)$ at such a point can be counted as a double zero, and Eq. (3) continues to be valid. Observe next that the difference function can be written as

$$
\begin{aligned}
\Delta \widehat{E}(\omega) & =\widehat{E}_{b}(\omega)-\widehat{E}_{a}(\omega) \\
& =W(\omega)[A(\omega)-B(\omega)] \\
& =W(\omega) \sum_{n=0}^{M}\left(a_{n}-b_{n}\right) \cos (n \omega)
\end{aligned}
$$

Note that the desired response $D(\omega)$ is cancelled when we take the difference between errors. Since $\cos (n \omega)$ can be written as a polynomial in $\cos \omega$, we can rewrite $\Delta \widehat{E}(\omega)$ as a polynomial in $\cos \omega$, that is,

$$
\Delta \widehat{E}(\omega)=W(\omega) \underbrace{\sum_{n=0}^{M} p_{n} \cos ^{n} \omega}_{P(\omega)}
$$

In the above equation, the cosine polynomial $P(\omega)$ is defined for all $\omega$. Even though the weight function $W(\omega)$ is defined only in the union of bands of interest, it can readily be defined in the transition bands to have some arbitrary positive values. Thus the expression (4) is defined for all $\omega$. And since $W(\omega)>0$, the zeros of $\Delta \widehat{E}(\omega)$ are precisely the set of zeros of the cosine polynomial $P(\omega)$. Summarizing, the number $N_{z}$ in Eq. (3) is nothing but the number of zeros of the polynomial $P(\omega)$. Next observe from the figure that the difference function $\Delta \widehat{E}(\omega)$ has a change of sign as we move from the last extremum in one band to the first extremum in the next band. For example, we see from Fig. 2(b) that

$$
\Delta \widehat{E}\left(\omega_{e_{1}}\right) \geq 0, \quad \Delta \widehat{E}\left(\omega_{e_{2}}\right) \leq 0
$$

where $\omega_{e_{1}}$ and $\omega_{e_{2}}$ are neighbouring extremal frequencies from adjacent bands.

(a)

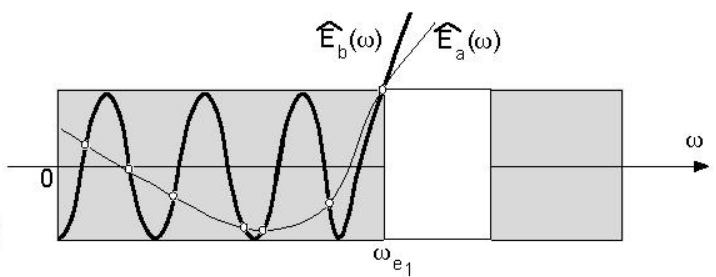

(b)

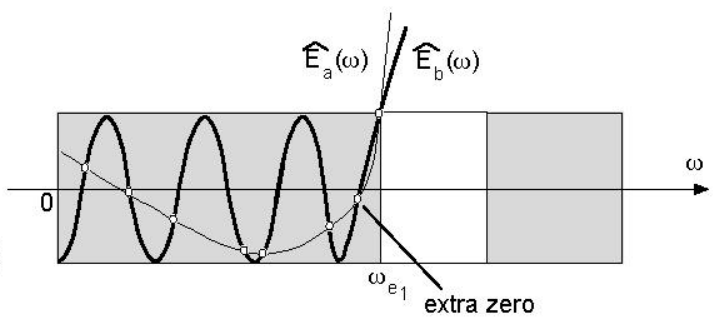

(c)

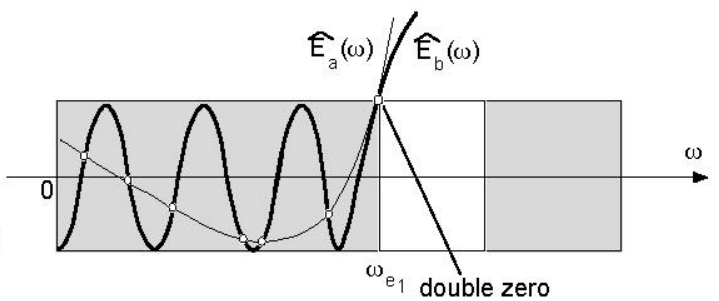

Fig. 3. (a)-(c) Pertaining to the sign of the difference $\widehat{E}_{b}(\omega)-$ $\widehat{E}_{a}(\omega)$. (a) Conventional cut, and (b), (c) less common cuts.

Assume first the inequalities in (5) are strict as in Fig. 2(b). Then the function $\Delta \widehat{E}(\omega)$ crosses zero when frequency increases from $\omega_{e_{1}}$ to $\omega_{e_{2}}$. In short, the difference $\Delta \widehat{E}(\omega)$ not only has zeros in the $K$ bands, it also has a zero in the space between the last extremum of a band and the first extremum of the next band. Thus the number of zeros $N_{z}$ of the cosine polynomial $P(\omega)$ in the frequency range $[0, \pi]$ is 
such that

$$
N_{z} \geq \sum_{k=1}^{K} N_{k}-K+K-1=\sum_{k=1}^{K} N_{k}-1 \geq M+1
$$

where the last inequality above follows from the assumption that are at least $M+2$ extrema (see (2)). But we know that a polynomial of the form $\sum_{n=0}^{M} p_{n} x^{n}$ has only $M$ zeros. Setting $x=\cos \omega$ and observing that $\cos \omega$ is monotonically decreasing in $0 \leq \omega \leq \pi$ we conclude that $P(\omega)$ cannot have more than $M$ zeros in $0 \leq \omega \leq \pi$. Equation (6) is therefore a contradiction of this unless $\bar{P}(\omega)$ is identically zero, that is, $p_{n}=0$ for all $n$. This means that $B(\omega)=A(\omega)$ indeed. Summarizing, the weighted equiripple solution with at least $M+2$ extrema is the unique solution which minimizes the maximum weighted error.

If the intersection of $\widehat{E}_{a}(\omega)$ and $\widehat{E}_{b}(\omega)$ occurs right at the band edge $\omega_{e_{1}}$ we have $\Delta \widehat{E}\left(\omega_{e_{1}}\right)=0$. See Fig. 3(a). However, since $P(\omega)$ is continuous, this still guarantees that $\Delta \widehat{E}\left(\omega_{e_{1}}+\epsilon\right)>0$ for sufficiently small $\epsilon$. Thus $\Delta \widehat{E}(\omega)$ still has a zero-crossing between the last extremum of one band and the first extremum of the next (in $\omega_{e_{1}}<\omega<\omega_{e_{2}}$ in the figure). The only exception to this argument would be the two situations shown in Figs. 3(b) and 3(c). One is when $E_{a}(\omega)$ cuts $E_{b}(\omega)$ from the "wrong side" as in Fig. 3 (b). In this case an extra intersection or zero is generated as demonstrated in the figure. The other is when the two error plots are tangential at $\omega_{e_{1}}$ (Fig. 3(c)) in which case we can count the zero of $\Delta \widehat{E}(\omega)$ at $\omega_{e_{1}}$ as a double zero. In either situation, therefore, the claim (6) continues to be true, and all arguments in the preceding paragraph continue to be valid. The proof is therefore complete.

$\nabla \nabla \nabla$

\section{CONCLUDING REMARKS}

We conclude the paper with a number of important observations pertaining to the above proof.

1) The proof in Sec. 2 uses the fact that there is a transition band between every pair of adjacent bands of interest. There could be more transition bands, one to the left of the leftmost band, and one to the right of the rightmost band, as demonstrated in Fig. 4. These bands do not affect the proof.

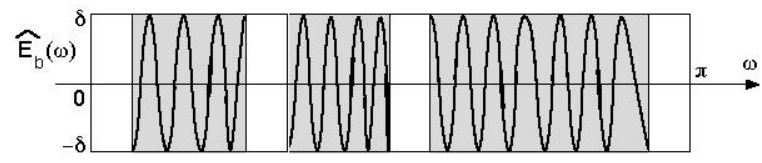

Fig. 4. An example with more transition bands (white areas) than desired bands (grey areas).
2) An example of a one-band approximation with two transition bands is the Type 3 Hilbert transformer [8] In this case the function $B(\omega)=\sum_{n=0}^{M} b_{n} \cos (\omega n)$ is required to approximate $1 / \sin \omega$ in the passband region $\omega_{1} \leq \omega \leq \omega_{2}$, where $\omega_{1}>0$ and $\omega_{2}<\pi$. See Fig. 5. The product $\sin \omega B(\omega)$ eventually turns out to be as in Fig. 5(b) so that it approximates unity in the above passband. The weighted error is $\widehat{E}(\omega)=$ $W(\omega)[D(\omega)-B(\omega)]$ where $D(\omega)=1 / \sin \omega$ and $W(\omega)=\sin \omega$ so that

$$
\widehat{E}(\omega)=1-\sin \omega B(\omega)=1-\sin \omega \sum_{n=0}^{M} b_{n} \cos (\omega n)
$$

Even though the derivative of $\sum_{n=0}^{M} b_{n} \cos (\omega n)$ can have only $M-1$ zeros in the passband, the derivative of the product

$$
\sin \omega \sum_{n=0}^{M} b_{n} \cos (\omega n)
$$

can have more, and the number of extrema including the band edges can indeed be as large as $M+2$ as required by the alternation theorem. For example suppose the Hibert transformer order is $N=52$ so that $M=(N-2) / 2=25$. Then the number of extrema in the plot, counting the bandedges, is indeed $M+2=27$, as can readily be verified by performing the design using the remez command in Matlab.

(a)

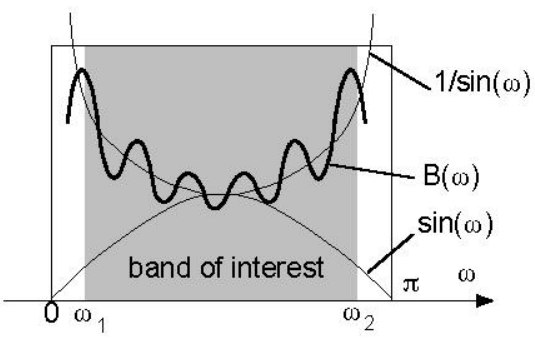

(b)

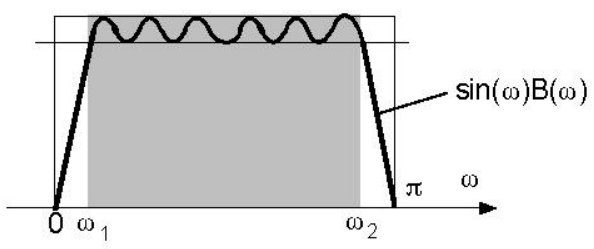

Fig. 5. Some details in the Hilbert transformer approximation.

As remarked in the beginning of Sec. 2, the existence of an optimal solution follows from compactness arguments [9]. As a thought for future, it will be nice to develop a "simple" 
proof for the existence of equiripple solutions, which can be presented at an introductory level to signal processing students.

\section{REFERENCES}

[1] A. Antoniou Digital signal processing, McGraw-Hill, 2006.

[2] E. W. Cheney, Introduction to approximation theory, McGrawHill Book Co., N. Y., 1982.

[3] J. H. McClellan, and T. W. Parks, "A unified approach to the design of optimum FIR linear phase digital filters," IEEE Trans. Circuit theory, vol. CT-20, pp. 697-701, Nov. 1973.

[4] S. K. Mitra, Digital signal processing, McGraw-Hill, 2006.

[5] A. V. Oppenheim and R. W. Schafer, Digital signal processing, Prentice Hall, Inc., 1975.

[6] A. V. Oppenheim and R. W. Schafer, Discrete time signal processing, Prentice Hall, Inc., 1999.

[7] J. G Proakis and D. G. Manolakis, Digital signal processing, Prentice Hall, 1996.

[8] L. R. Rabiner and B. Gold, Theory and application of digital signal processing, Prentice Hall, Inc., 1975.

[9] J. R. Rice, The approximation of functions, Addison-Wesley Publ. Co., Reading, MA, 1964. 